\title{
Price effect analysis and pre-reseravtion scheme on electric vehicle charging networks
}

\author{
Junghoon Lee, Gyung-Leen Park \\ Department of Computer Science and Statistics, Jeju National University, Republic of Korea
}

\begin{tabular}{l}
\hline \hline Article Info \\
\hline Article history: \\
Received Mar 1, 2019 \\
Revised Jul 21, 2019 \\
Accepted Jul 28, 2019 \\
\hline
\end{tabular}

\section{Keywords:}

Charging network

Electric vehicle

Pre-reservation

Price effect

Revenue increase

\begin{abstract}
This paper investigates the price effect to the charging demand coming from electric vehicles and then evaluates the performance of a pre-reservation mechanism using the real-life demand patterns. On the charging network in Jeju city, the occupancy rates for 3 price groups, namely, free, medium-price, and expensive chargers, are separated almost evenly by about $9.0 \%$, while a set of chargers dominates the charging demand during hot hours. The virtual pre-reservation scheme matches electric vehicles to a time slot of a charger so as not only to avoid intolerable waiting time in charging stations systematically but also to increase the revenue of service providers, taking into account both bidding levels specified by electric vehicles and preference criteria defined by chargers. The performance analysis results obtained by prototype implementation show that the proposed pre-reservation mechanism improves the revenue of service providers by up to $9.5 \%$ and $42.9 \%$, compared with the legacy FCFS and reservation-less walk-in schemes for the given performance parameter sets.
\end{abstract}

Copyright () 2019 Institute of Advanced Engineering and Science. All rights reserved.

\section{Corresponding Author:}

Gyung-Leen Park,

Department of Computer Science and Statistics,

Jeju National University,

Jejudaehakno 102, Republic of Korea.

Email: jhlee@jejunu.ac.kr

\section{INTRODUCTION}

Electric vehicles, or EVs in short, are replacing legacy gasoline-powered vehicles, bringing a pollution-free transport era [1]. As they get energy from the electricity network, transport systems are becoming a part of the electricity grid, specifically, the smart grid [2]. Definitely, the wide expansion of charging infrastructures, including charging stations, energy provisioning, and the like, is the most important prerequisite for the fast penetration of EVs. However, we cannot completely avoid the insufficiency in the charging capacity or the demand-supply mismatch in early stages. Hence, it is necessary to employ an efficient management system for charging facilities to monitor their real-time behaviors, not only to detect failures immediately but also to obtain operation records for better planning. Moreover, the management system can support a reservation mechanism and also implement an appropriate price policy based on the analysis of the operation history data [3].

Management capabilities, combined with a reservation strategy, can alleviate inherent inconvenience stemmed from long charging time by freeing drivers from wasting their time in the waiting queue [4]. Moreover, a sophisticated matching mechanism between EVs and chargers can be designed to distribute energy load over the time and space axis, as well as to increase the profit of charging service providers [5]. It is true that the charging cost has less effect on the charging demand, compared with gasoline fueling, due to much cheaper electricity price. However, local drivers, who are familiar with the current charging prices around their vicinity, have no reason to disregard the price difference. This allows us to shape the overall demand by lowering the charging fee on non-busy chargers during the hours of low traffic. 
In addition, if the charging price is set higher for the hot stations during peak hours, the revenue of charging service providers will increase [6].

The first step for price policy planning is the analysis of the cost effect to the charging demand. It can be carried out making use of real-life operation records accumulated in the target EV charging system. Our city, namely, Jeju, Republic of Korea, currently runs a citywide charging network consisting of about 245 DC chargers installed for public use [7]. There are 4 charging service providers from both civil and governmental domains, while the national authority makes them report their real-time working status to the central server via the available communication channel. After acquiring the permit to exploit this data, our application retrieves the operation records of chargers belonging to our city from the central database every 5 minutes and stores in the local database, creating an evergrowing time-series. It must be mentioned that chargers are added or detached to or from the monitoring system for many reasons such as new installations, component failures, operation strategy changes, and the like. Therefore, the number of chargers, even not so frequently, keeps changing and the dataset undergoes instability from time to time.

In the meantime, we can assume that a pre-reservation application for the next day charging will appear with the support of hardware implementation which directly controls the charger operation under the coordination of upper layer applications. Instead of processing reservation requests one by one, it allocates (charger, time slot) pairs to multiple EVs simultaneously to achieve the given performance goal. Then, it is possible to design a bidding mechanism for hot chargers during peak hours so as to avoid unpredictable energy demand imbalance. Actually, our research team has proposed an EV-charger matching scheme which not only increases the number of matches but also enhances the revenue of charging service providers, tailoring the stable marriage problem to take as many high-bidders as possible [8]. However, the performance has been measured using a rather unrealistic random process model. Hence, this paper is to first summarize the price effect on charging demand using the Jeju dataset [9] and then feed the result to the EV-charger pre-reservation mechanism to assess its performance based on a real-life scenario.

This paper is organized as follows: After describing the main topic and its correlation with our previous researches in Section 1, Section 2 shows the main feature of the monitoring data archive and the analysis result in terms of the charging demand dynamics. Section 3 captures the realistic demand pattern for the evaluation of EV-related applications, beginning with the explanation of our pre-reservation mechanism. Section 4 conducts experiments to measure the performance of the EV-charging matching scheme with the analyzed charging load. Finally, Section 5 concludes this paper with a brief introduction of future work.

\section{PRICE EFFECT ANALYSIS}

\subsection{Data archive}

To begin with, we plot the location of each charger on the road network of Jeju city as shown in Figure 1. This map is downloaded from an open data site in an ESRI shape file format and plotted on the $\mathrm{R}$ package workspace [10]. The coastline of this island stretches about $200 \mathrm{~km}$ long, while the two most populated towns are located in the northernmost and southernmost regions. Jeju city is one of the most famous tourist attractions in the East Asia, so tourists occupy a large portion of the whole city traffic almost all year round. Therefore, the charging load is not restricted to local residents but comes from tourists driving EV rent-a-cars. The figure shows that chargers are installed largely in proportion to the population density, which is definitely interrelated with the current EV penetration [7]. Thus, we can see two dark areas in the upper and lower parts of the map. In addition, to meet the demand from tourists, some chargers are installed in famous tourist attractions. Those chargers are scattered over the city area. In the figure, when more than one charger is installed in the same building, multiple letters overlap and just one appears.

On the map, chargers are marked by F (Free), M (Medium-price), and E (Expensive), respectively. There are currently 3 price levels. First, it is free (F) on 60 chargers operated by the local government. Second, it costs about 3 USD (M) to fully charge a $20 \mathrm{kwh}$ EV battery on 49 chargers possessed by a national government ministry. Last, 6 USD (E) is charged for the same electricity amount at 136 chargers run by private service providers [7]. Even though the diversity of price levels is quite limited, we can estimate how much the price will influence the driverside behavior for future charger deployment. As mentioned earlier, the number of chargers under the control of the monitoring system has changed from time to time. During August 2017, the charging network most reliably operated, andling the largest number of charging transactions. Hence, this research focuses on the data archive obtained during this period. Besides, slow AC chargers, mainly installed in individual homes for overnight charging, are not included in the monitoring domain, as they have no reason to be globally coordinated. We are only interested in the public fast chargers. 


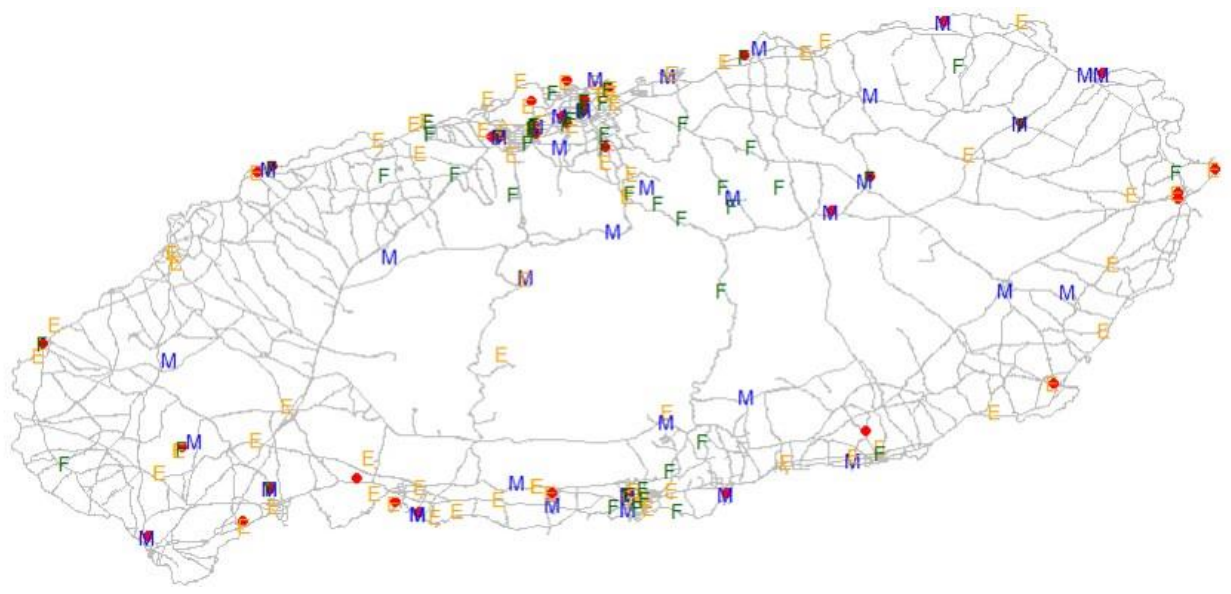

Figure 1. EV charger distribution

This data archive is quite different from the legacy ones which take charging records from respective Evs [11]. So, we select the occupancy rate as the main metric for the price effect analysis in our experiment. Here, the occupancy rate is the probability that a charger is occupied at a specific time instant and calculated by dividing the number of reports indicating a charger is servicing an EV by that of total reports. In case a new EV replaces the previous one within a 5-minute report interval in the same charger, it is not possible to know whether a new transaction has started, as the report does not include EV identifiers for the sake of privacy protection. Hence, the occupancy rate is the most meaningful information we can get with this data archive. It gives us an insight of charger utilization and charging demand. Based on the above definitions, one of our previous works clusters the daily pattern on each charger to check if there exists a common service behavior shared by a group of chargers, but without the consideration of the price level [7].

\subsection{Demand behaviors}

The first experiment measures the occupancy rate for those 3 groups of chargers during August 2017, and the results are shown in Figure 2. Figure 2(a) plots the occupancy rate for a whole day, while Figure 2(b) only for the period from 12 PM to 6 PM. Charging demand is concentrated in this interval, during which the citywide energy demand also reaches the peak. Figure 2(a) shows that the occupancy rate of free chargers is higher than the others by 8.9 and $9.3 \%$ on average, respectively, while the occupancy rate difference between medium-price and expensive chargers is very small. On the contrary, the gaps between 3 curves becomes more vivid in Figure 2(b). Free chargers are more frequently used than medium-price chargers by up to $13.0 \%$, but the gap has been once reversed. This situation can possibly happen, as more medium-price chargers are installed in tourist attractions, where the daytime demand can explode. Anyway, the enlarged gap indicates that some drivers change chargers when finding that free ones they want are already occupied in busy hours. However, even in that case, they rarely choose expensive ones.

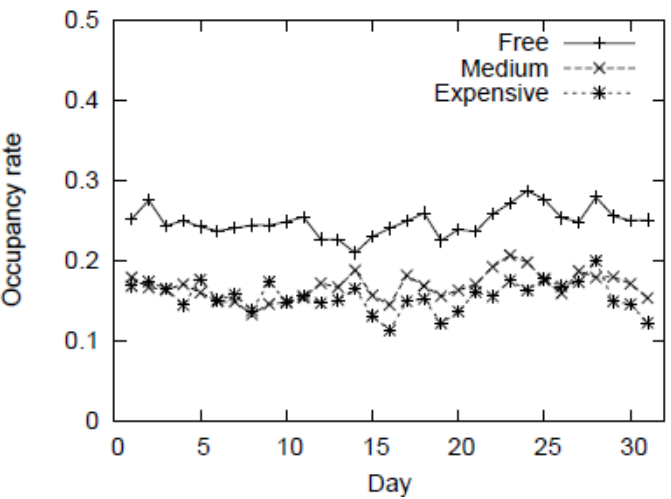

(a) Whole day occupancy rate

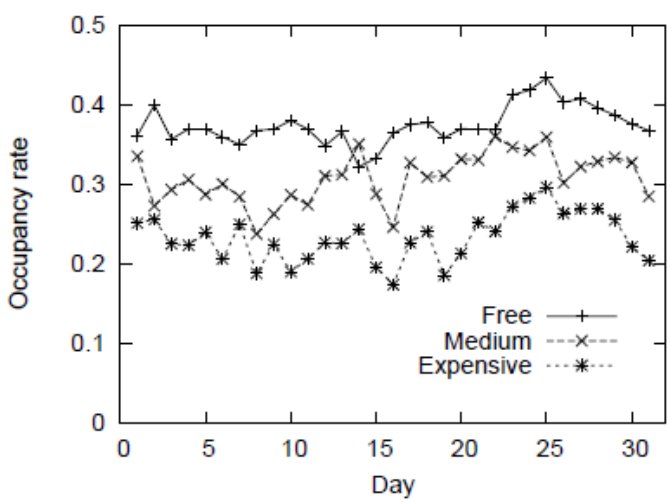

(b) Hot-hour occupancy rate

Figure 2. Daily charging behavior 
Next, Figure 3(a) and Figure 3(b) plot the hourly occupancy rates for all chargers and those for hot 20 chargers, respectively, to check the load imbalance between the chargers. Our observation has discovered that about 50 chargers dominate the overall charging activities. Among these, 23 free, 12 medium-price, and 15 expensive chargers are included and they are usually installed in high-population areas. Most of all, the two figures show an absolute difference in the occupancy rate. Interestingly, expensive chargers are more frequently used than the others over the time interval from 2 AM to 4 AM. Drivers seem to charge their EVs without considering the price at dawn. On the contrary, during daytime, the difference between the three is magnified. The peak demand takes place between 16 PM and 18 PM in Jeju, where tourists occupy a large portion of citywide traffic on the road. Intuitively, daily tours usually need one more charging around this time, provided that EVs are fully charged overnight and start their trips in the morning. In addition, Figure 3(b) traces individual demand patterns. Here, 20 out of hot 50 chargers are randomly selected to avoid too much curve complexity. The figure shows that most of high-demand chargers share a common demand pattern, indicating that a price policy can be applied uniformly. After all, a reservation mechanism is necessary during the interval from 12 to $6 \mathrm{PM}$.

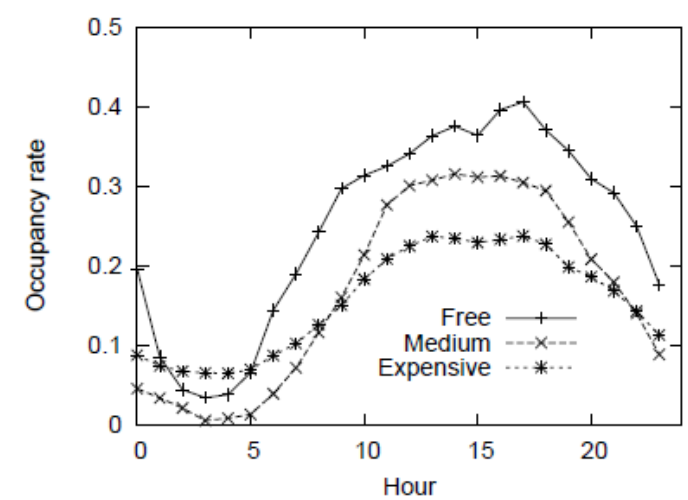

(a) Hourly occupancy rate

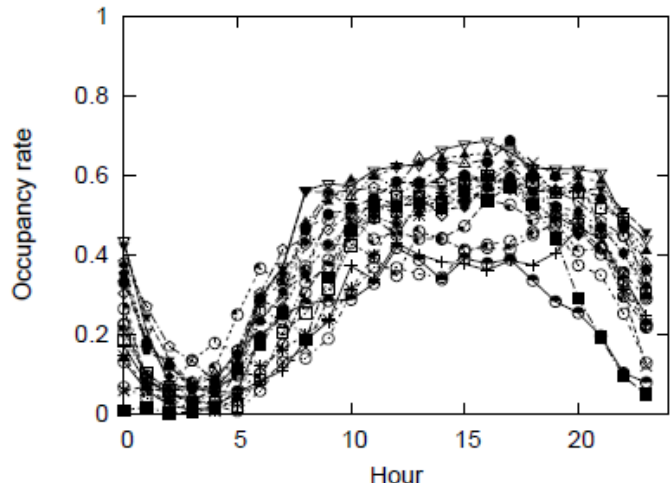

(b) Hot-charger hourly rate

Figure 3. Hourly charging behavior

As an extended version of [9], this work focuses on the price effect to the demand pattern to observe the performance of EV charging applications. For more detailed experiment results, including the effect of charger density and the distance to a nearby free charger to the occupancy rate, refer to [9]. In addition, from the temporal on-off stream, we can detect the start and end time of charging transactions. It allows us to estimate the length of charging time, the amount of energy, and the like, much reducing the amount of memory to store the data archive.

\section{DEMAND PATTERN FOR THE PRE-RESERVATION MECHANISM}

\subsection{Reservation mechanism}

Without a reservation mechanism, an EV driver visits a charging station on the route and takes a charger if available. Otherwise, the driver will either wait or move to another station. Even though a relevant application shows that a charger is free now, it can be taken by other EVs, while an EV is heading for the charger. As the service time, namely, the charging transaction length, for each EV can extend to tens of minutes, the waiting time can grow soon. With the support of an interface circuit capable of injecting electricity to an EV during the specific time interval under the control of the coordination entity, a charging station can provide a reservation mechanism. Generally, a reservation request is processed one by one according to the FCFS (First Come First Service) policy. Here, the request submitted first has an ultimate priority over the latter ones, while drivers select chargers (along with time slots) out of available ones with the help of an application which shows the up-to-date reservation status [12]. An EV can issue a reservation request until just before the time slot it wants, as long as it can arrive.

Even though the FCFS policy is so common, easy to implement, and fair to requesters, it cannot coordinate requests to achieve other system goals such as global peak shaping and personalized pricing. Meanwhile, there are EVs whose next day tour schedules are fixed, for example, delivery services, tourists vehicles, and scheduled business trips. Moreover, EVs can possibly try to reserve a charger and make a tour plan according to the reservation result, when they have multiple options. In this case, a set of requests from 
EVs are handled simultaneously. That is, the coordinator collects requests by the end of a certain deadline and pairs EVs and chargers. Here, an EV may submit more than one charger it wants, while at most one can be admitted. Then, many-to-many coordination is carried out in a batch mode as a variant of bipartite matching. This is the main idea of pre-reservation. We assume that the time axis is divided into a series of fixed-size slots and reservations are made by the slot. EV charging can start only from the beginning of the time slot the EV has reserved.

From the viewpoint of EVs, the reservation is fulfilled in two steps, namely, pre-reservation and ordinary FCFS-based reservation, respectively. In the pre-reservation step, EVs can basically nominate multiple (charger, slot) pairs they want. The reservation results are not created until the end of an application deadline. Then, slots are matched to EVs according to the preference at the EV side as well as the priority function set by chargers. An EV may fail in reserving a slot if all request entries are beaten by others. A slot cannot be assigned in case no EV has applied. Those EVs failed in the pre-reservation stage are required to follow the next reservation process. Here, newcomers can join the process and each request is handled one by one. Here, participants do not have to name multiple candidates simultaneously. In the second step, the driver must search slots left after the pre-reservation process and select the one which best meets his or her driving schedule. We think that some Internet portals provide web services indicating the location of chargers on the map and can possibly implement a reservation mechanism just like hotel booking services. Our design puts emphasis on the pre-reservation step, as the ordinary process is not peculiar and has nothing to improve.

Figure 4 summarizes this mechanism, assuming that $n$ chargers are participating for the time period from $12 \mathrm{PM}$ to $18 \mathrm{PM}$. Only during the hot hours, the reservation service is provided, while not every charger participates in the service. The time slot is 30 minutes, making the total number of slots to match is $n \times 12$ if each charger opens 12 slots for reservation. The slot length will be roughly set to the average charging transaction time and must include the overhead to manipulate the registration device, plug the EV connector into a charger, pay the fee, and the like. We assume that EVs will leave before the end of reserved time slots, possibly due to strong penalty policies. In the figure, slots are numbered sequentially from Charger 0 . An EV specifies the lists of (charger, slot) pairs, which are mapped to respective identifiers for allocation. As at most one of the requests can be accepted per EV, the slot assignment is reduced to a bipartite matching problem.

Without any other requirement, the goal of the match procedure will be to maximize the number of (EV, slot) pairs. However, chargers prefer highest bidders to improve their revenue. Then, there are weights on EV-slot links and the problem gets complex. Here, the preference from each party is explicitly defined. On the EV side, each EV simply lists (charger, slot) pairs according to its preference order. Unlike the ordinary matching scheme, the number of application entries is much smaller than the total number of slots and can be different EV by EV. On the other hand, at the charger side, a charger gives priority to those EVs which want to pay more to increase its revenue. To this end, our service makes EVs submit the price they would like to pay, making a reservation entry (charger, slot, bid). In the example of Figure 4, EV 0 applies for 2 slots while EV 1 just one. Then, the reservation mechanism chooses first those requests bidding higher. In case two or more biddings are tied, the request having a shorter preference length will be selected as it has less possibility to be picked. If tied again, the requests which arrived first will be preferred. After all, the preference function at the charger side is completed. This procedure is customized from the SMP (Stable Marriage Problem) solver.
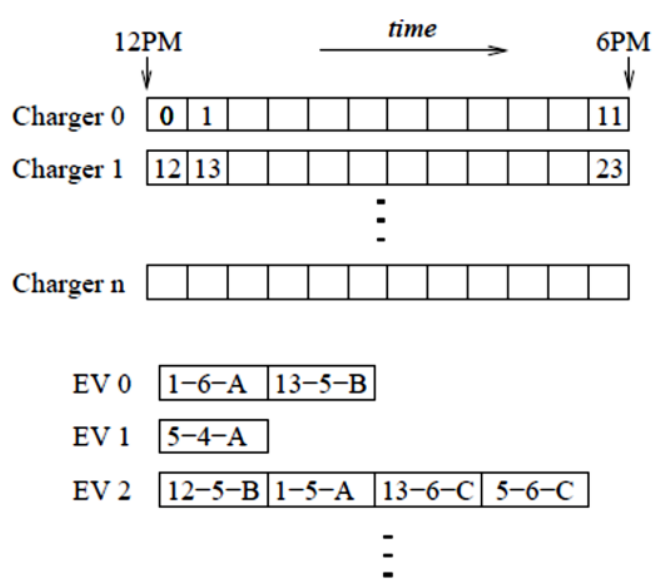

Figure 4. Matching-based pre-allocation 
The bipartite matching procedure runs with the class definition shown in Figure 5 [8]. The Charger class is instantiated for each (charger, slot) pair. Each EV object contains a preference list consisting of multiple request entries, while pos field points to the request entry to process next. It cannot go beyond the number of entries and if it does, the EV cannot be assigned to a slot and will be eliminated from the matching process. In addition, the match field in the charger class keeps track of the current match. The matching scheme processes from the first EV, which arrives first. However, this order does not affect the final matching result. If the (charger, slot) is not yet matched, it will be assigned to the EV, that is, the match field in the charger class will be set to that EV. Otherwise, the current EV and the challenging EV fight according to the criteria described previously. The winner will take the place while the loser will continue the contention with the next request entry. Here, the pos pointer will proceed by one. We do not consider the case a single EV tries to reserve two or more slots simultaneously, as one full charging can cover daily trips in most cases.

\begin{tabular}{|l|l|}
\multicolumn{1}{c|}{ Charger class } & \multicolumn{2}{c|}{ EV class } \\
\hline $\begin{array}{l}\text { id } \\
\text { match }\end{array}$ & $\begin{array}{l}\text { id } \\
\text { preference list } \\
\text { (charger, slot, bid) } \\
\text { pos }\end{array}$ \\
\hline $\begin{array}{l}\text { Preference Function } \\
\text { 1) bidding class } \\
\text { 2) EV pref. length } \\
\text { 3) arrival time }\end{array}$ \\
\hline
\end{tabular}

Figure 5. EV and charger preference

\subsection{Request generation scenario}

As shown in the previous section, each charger has its own different occupancy rate, and we think that this demand distribution will be kept as life styles or driving patterns of citizens will not change in a short time. The number of chargers which will participate in the reservation service is not known yet. However, for those in the service group, EVs will send reservation requests much similarly as the current demand pattern. Hence, for $n$ chargers participating in the reservation service, our load generation module sums up the current occupancy rates for them and then calculates the probability that a charger will be selected just like the well-known Roulette wheel method. Here, $n$ is given as an experiment parameter. As for the number of EVs, EVs will be mainly bought by local residents in the downtown area due to easy accessibility to charging facilities. Additionally, EVs will be purchased by rent-a-car companies servicing for tourists, who get more interested in eco-friendly driving with EVs. Even though the number of EVs taken by tourists gets higher from time to time, especially during the vacation seasons, the majority of EVs on the road is owned by locals. Hence, the charging demand will not change in spite of forthcoming penetrations.

Next, the probability that an EV selects low-price chargers can be inferred from the current occupancy rate according to the price level. The drivers actively preferring free chargers are sensitive to the charging cost. Roughly speaking, the demand ratio for 3 groups is 2:3:4, each of which represents expensive, medium-price, and free chargers, respectively. As the price level will be different and keep changing, especially when combined with the bidding process in the near future, there exists a driver group which will put the top priority on the charging cost and will bid as low as possible. Moreover, locals tend to more like cheaper chargers. Hence, we set the ratio of the group as a performance parameter. In the data archive analysis, the difference in utilization between medium-price and expensive chargers gets less vivid along the time axis. The selection seems to depend more on the charger location than the cost. Hence, for the non-sensitive group, the probability to select out of the rest of chargers, will be the same, namely, distributes randomly.

\section{PERFORMANCE ANALYSIS RESULTS}

This section measures the performance of the proposed scheme, comparing with the legacy FCFS strategy and the reservation-less walk-in policy. The performance metrics include the number of matched charges and the gained profit. The experiment takes number of EVs, number of chargers (slots), average preference length, and price sensitivity as performance parameters. By default, they are set to 800, 50, 3.0, and 0.33 , respectively. Performance is measured changing one parameter with the others remaining at default 
values. Our prototype is implemented with the Java programming language and its execution time is observed to be less than 0.1 seconds for all slot assignments, making the reservation process a real-life application. That is, an average performance PC can run the pre-reservation process for thousands of EVs and ten thousands of slots within an acceptable time bound. The request entries, namely, (charger, slot, bid) tuples, will be generated according to the load analysis result obtained in the previous section. In addition, for all parameter settings, 20 sets are generated and the obtained results are averaged.

The first experiment measures the effect of the number of EVs, and the results are shown in Figure 6. First, Figure 6(a) plots the number of accepted requests, when the number of EVs participating in the matching process ranges from 100 to 1,000 . Here, the length of a preference list ranges randomly from 1 to 5, making the average converge to 3.0. As can be seen in the figure, the curve of the proposed SMP-based scheme outperforms the other two. However, the gap from the FCFS scheme is not so large, at most $6.5 \%$, for 500 EVs. This enhancement is achieved as the SMP-based scheme gives precedence to EVs having shorter preference length. At the two range boundaries, the gaps are below $1.0 \%$. Even though the improvement is quite small, the proposed scheme works better for the whole experiment settings. The performance gap from the walk-in scheme reaches $27.8 \%$ when the number of Evs is 600 . The number of available slots is also 600, so for the current demand distribution, about 1:1 EV-charger ratio can enhance the reservation efficiency.

Next, Figure 6(b) shows the gained profit for 3 schemes. Here, we assume that there are 3 bidding classes, each of which costs 6,5 , and 4 USD, respectively, for a full slot occupation. This selection considers the current charging price policy in Jeju. We assume that the price is independent of the amount of charged electricity, as most Evs making reservations highly likely to want to charge almost to the full level. The proposed scheme gives precedence to those requests bidding higher. The number of bidding levels can get larger and so does the price gap between bidding classes. However, how to set those parameters is another problem. The figure shows that the gained profit increases by $32.3 \%$ and $9.5 \%$, compared with the walk-in and FCFS schemes, respectively. The earned profit gets higher when the number of EVs gets closer to the number of (charger, slot) pairs. Actually, in the real-life request model, charging demand is more concentrated to a small set of chargers. The performance enhancement is relatively smaller than the case of random charger selections as in [8].

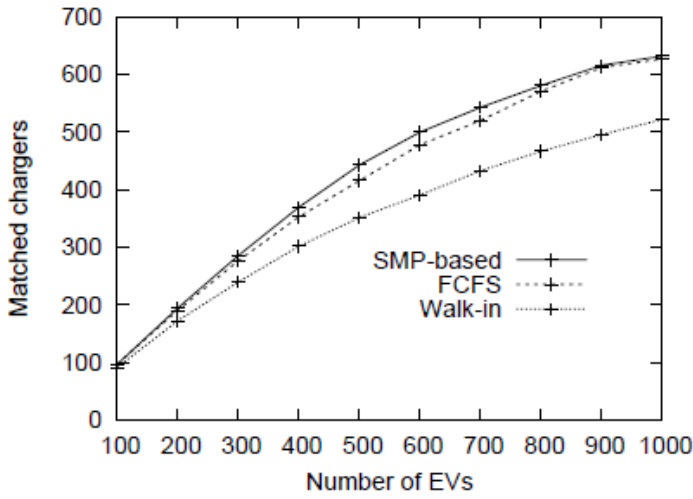

(a) Matched chargers

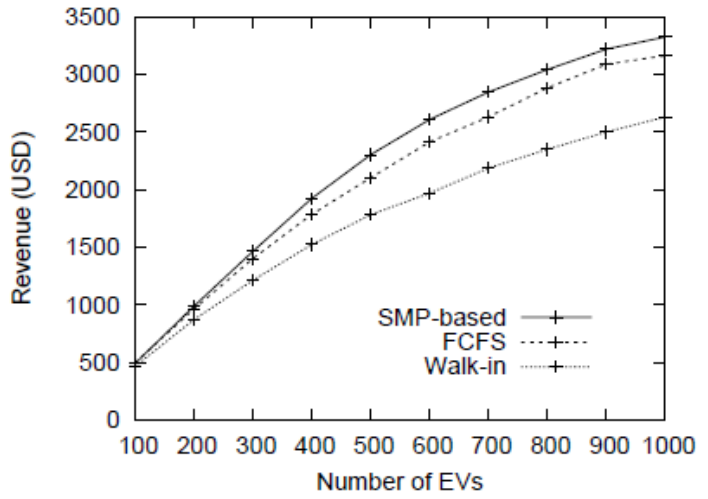

(b) Gained profit

Figure 6. Effect of the number of EVs

Figure 7 plots the effect of the number of chargers, and the experiment makes it range from 20 to 80 . This means that the number of slots ranges from 240 to 960 . Here, EVs select a (charger, slot) according to the current load distribution. If the number of chargers is 20 , it means that top 20 chargers in the occupancy rate are included in the service group. Hence, when the number of chargers increases, the demand imbalance also gets higher. As shown in Figure 7(a) plotting the number of matched requests, the SMP-based scheme outperforms the others except one parameter set. The benefit gained from giving precedence to a shorter preference list diminishes in this situation. The proposed scheme increases the number of matched pairs by up to $24.6 \%$, compared with the walk-in scheme. In addition, the gained profit curve is quite similar to the case of Figure 6(b). The SMP-based scheme improves the revenue by up to $6.5 \%$ and $31.8 \%$, compared with the FCFS and the walk-in schemes. The performance gap tends to get enlarged according to the increase in the number of chargers as more options are available in matching slots. 


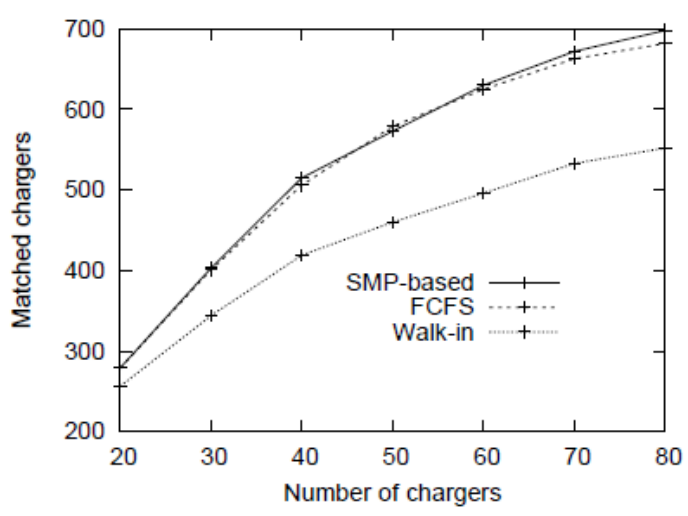

(a) Matched chargers

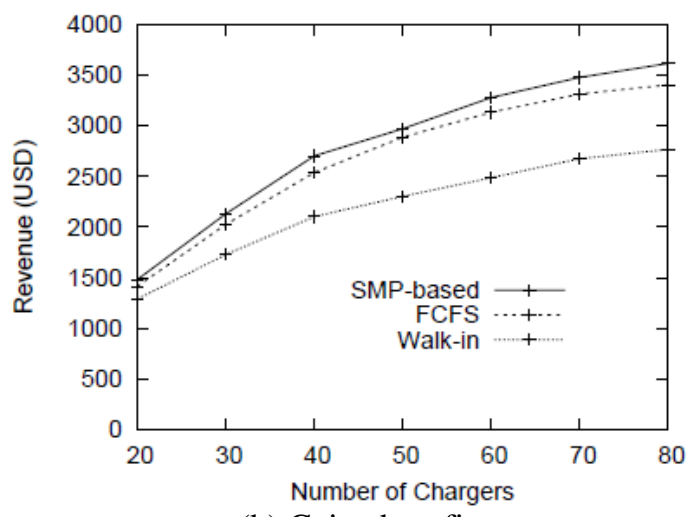

(b) Gained profit

Figure 7. Effect of the number of chargers

Now, the experiment measures the effect of the average length of the preference list, and the results are shown in Figure 8. The longer a preference list, the more options the reservation mechanism can have. Figure 8(a) shows the number of matched charges when the average number of preference list length ranges from 1.5 to 5.0. To begin with, the number of matched requests is almost same for SMP and FCFS-based schemes, and there is no superiority between them. Even though the SMP-based scheme more matches EVs and chargers in total, the gap is less than $2 \%$, compared with the FCFS scheme. The FCFS scheme matches more in 3 out of 7 parameter selections. It tells that both have the same chance for the number of matched pairs. When the preference list is sufficiently large and the FCFS scheme can also take advantage of plenty selectable options. The reservationless scheme is not influenced by the list length, as it takes a charger or waits until the charger becomes available. The SMP-based scheme outperforms the walk-in scheme by up to $33.8 \%$. The performance gap gets larger according to the increase in the number of charger tuples in the list.

In addition, Figure 8(b) shows the gained profit according to the preference list length. Even though the number of matched charges is almost same, this result reveals that our scheme improves the revenue of charging service providers by $7.2 \%$, compared with the FCFS scheme. As expected, the gap gets larger according to the increase in the list length, benefiting from the large option space. Besides, our scheme earns $42.9 \%$ more revenue than the walk-in scheme. While the FCFS scheme can also benefit from a larger preference list in the number of matched pairs, the SMP-based scheme can select higher bidders more efficiently. However, it is quite unrealistic for EVs to list more than 5 candidates for the next day tour schedule. It is desirable to give an additional benefit, such as higher discount rate, to EVs having a larger list, as they can enhance the possibility of successful matching. Actually, the pre-reservation needs to offer an early bird rate, cheaper than the regular reservation or walk-in charging.

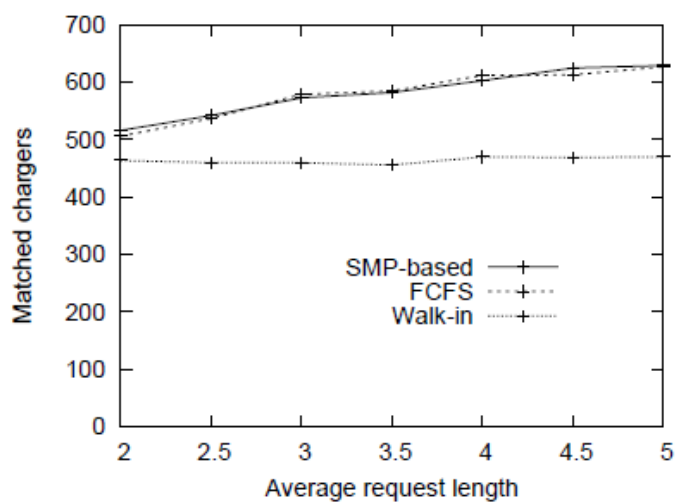

(a) Matched chargers

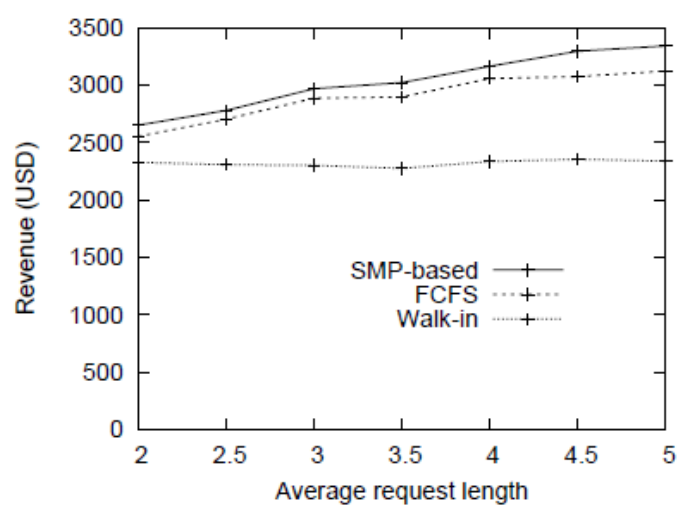

(b) Gained profit

Figure 8. Effect of the preference length 
Finally, Figure 9 shows the effect of the price sensitivity. If it is 0 , all EVs, having no concern on the price, bid for the highest level. On the contrary, if it is 1, all EVs bid lowest. In these two extreme cases, there is almost no possibility for the proposed scheme to improve the revenue. The improvement can be obtained only by the increased number of matched pairs. For $p$ sensitivity, the two highest bidding levels are selected with probability of (1-p). Both have the same chance to be selected within this range. In Figure 9(a), the SMP-based and FCFS schemes show the almost same number of matched pairs, outperforming the walkin scheme by about $23 \%$ over the whole range. Next, as shown in Figure 9(b), the SMP-based scheme improves the revenue by up to $4.7 \%$ compared with the FCFS scheme, when the sensitivity is 0.5 . That is, EVs bid uniformly over the available bidding range. In addition, at both tips, the SMP-based scheme matches more pairs, showing a better revenue for the whole experiment range.

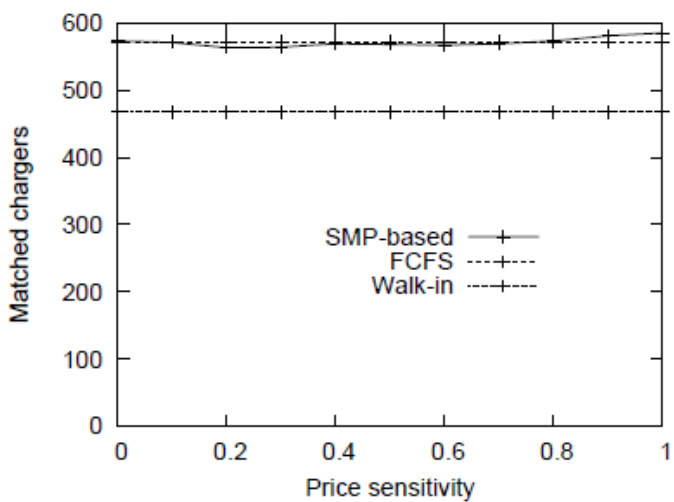

(a) Matched chargers

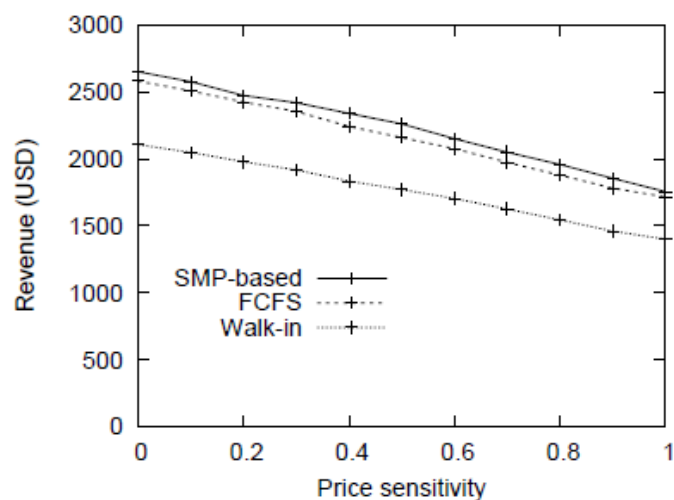

(b) Gained profit

Figure 9. Effect of the price sensitivity

\section{CONCLUDING REMARKS}

In this paper, we have analyzed the price effect to the charging demand taking advantage of data archives obtained from a charging network currently in operation. The occupancy rates for 3 price levels are evenly separated by $9 \%$, showing the linear price sensitivity. Hourly trace reveals the necessity of an efficient reservation mechanism for hot chargers during peak load period. The SMP-based pre-reservation scheme gives precedence to those EVs bidding higher, having a shorter preference list, and arriving first, sequentially. According to the performance measurement result using the practical demand model, the proposed pre-reservation mechanism improves the revenue of service providers by up to $9.5 \%$ and $42.9 \%$, compared with the legacy FCFS and reservation-less walk-in schemes for the given performance parameter sets. EV penetration is not mature yet and charging infrastructures are still under construction in many countries. Intelligent information technologies, especially sophisticated algorithms and artificial intelligence techniques, make it possible to overcome the current insufficiency and inconvenience in EV driving.

As future work, we will refine our demand model with the accumulation of charging operation archives and investigate the effect of new facility installation and EV deployment. This analysis will help us to design diverse EV applications such as new charger site selection [13], V2G (Vehicle-to-Grid) coordination [14], and the like. Moreover, renewable energy, such as wind and sunlight, has been drawing much attention in the target area. Our research team is planning to develop a citywide charging coordination integrating the renewable energy generation. Here, a variety of datasets are collected regarding solar energy generation and climate history records [15].

\section{ACKNOWLEDGEMENTS}

This research was supported by the 2019 scientific promotion program funded by Jeju National University, South Korea. 


\section{REFERENCES}

[1] S. Ramchrun, et al., "Putting the 'Smarts' into the Smart Grid: A Grand Challenge for Artificial Intelligence," Communication of the ACM, vol. 55, pp. 89-97, 2012.

[2] X. He, et al., "A big data architecture design for smart grids based on random matrix theory," IEEE Transactions on Smart Grid, vol. 8, pp. 674-684, 2017.

[3] J. Mukherjee and J. A. Gupta, "A review of charge scheduling of electric vehicles in smart grid," IEEE Systems Journal, vol. 8, pp. 1541-1553, 2014.

[4] S. Orcioni, et al., "Electric vehicles charging reservation based on OCPP," IEEE International Conference on Environment and Electrical Engineering, 2018.

[5] Z. Cao, et al., "An autonomous electric vehicle based charging system: Matching and charging strategy," IEEE Power \& Energy Society Innovative Smart Grid Technologies Conference, 2018.

[6] I. Bayram, et al., "A Survey on Energy Trading in Smart Grid," IEEE Global Conference on Signal and Information Processing, pp. 258-262, 2014.

[7] J. Lee and G. Park, "Temporal data stream analysis for EV charging infrastructure in Jeju," ACM Research in Applied Computation Symposium, pp. 36-39, 2017.

[8] J. Lee and G. Park, "Charger reservation scheme for electric vehicles based on stable marriage problem adaptation," International Conference on Information Technology: IoT and Smart City, 2018.

[9] J. Lee and G. Park, "Price effect analysis on electric vehicle charging networks in Jeju city," International Conference on Control, Mechatronics and Automation, pp. 175-179, 2018.

[10] C. Brunsdon and L. Comber, An Introduction to R for Spatial Analysis \& Mapping, SAGE Publication Ltd., 2015.

[11] J. Q. Torros, et al., "A statistical analysis of EV charging behavior in the UK," IEEE PES Innovative Smart Grid Technologies Latin America, pp. 445-449, 2015.

[12] A. Ghosh and Y. Aggrawal, "Menu-based pricing for charging of electric vehicles with vehicle-to-grid service," IEEE Transactions on Vehicular Technology, vol. 67, pp. 10268-10280, 2018.

[13] N. Shahraki, et al., "Optimal location of electric public charging stations using real world vehicle travel patterns," Transportation Research Part D, vol. 41, pp. 165-176, 2015.

[14] G. Buja, et al., "Reactive power compensation capabilities of V2G-enabled electric vehicles," IEEE Transactions on Power Electronics, vol. 32, pp. 9447-9459, 2017.

[15] B. Banhthasit, et al., "Optimal generation scheduling of power system for maximum renewable energy harvesting and power losses minimization," International Journal of Electrical and Computer Engineering (IJECE), vol. 8(4), pp. 1954-1966, 2018. 